\title{
In Their Own Words: Why Teenagers Don't Use Social Networking Sites
}

\author{
Rosland K. Baker, M.Psych., and Katherine M. White, Ph.D.
}

\begin{abstract}
We explored common reasons for non-use of the rapidly growing popularity of social networking sites among a sample of Australian adolescents $(N=69)$. Transcripts were coded by grouping responses along similar themes for non-use that had been commonly stated by participants. The primary reasons offered by adolescents were: lack of motivation, poor use of time, preference for other forms of communication, preference for engaging in other activities, cybersafety concerns, and a dislike of self-presentation online. The identification of these themes allows for a greater understanding of teenagers' decisions not to engage in the popular medium of communication and points to possible strategies that could be utilised by Web site developers in efforts to appeal to a wider teenage audience.
\end{abstract}

\section{Introduction}

$\mathbf{O}$ NLINE SOCIAL NETWORKING SITES (SNSs), such as MySpace and Facebook, are web-based services that allow individuals to construct public or semi-public profiles, connect with other users, and view and traverse their list of connections and those made by others within the system. ${ }^{1}$ Although there are currently no reliable data regarding how many people use SNSs, ${ }^{1}$ marketing research indicates that SNSs are growing exponentially in popularity worldwide. ${ }^{2}$ Further, SNSs have quickly become one of the leading communication tools for adolescents' online socializing, ${ }^{3}$ with some studies indicating that more than half of all online Australian adolescents use SNSs. ${ }^{4}$ Consequently, adolescents' engagement with these sites is increasingly attracting the attention of academic and industry researchers alike wishing to examine the benefits and concerns associated with SNS use.

Some of the noted benefits associated with SNS use include new opportunities for self-expression, sociability, community engagement, creativity, and new literacies. ${ }^{5}$ Conversely, some noted concerns include excessive use leading to reduced time for other activities, including academic, ${ }^{6}$ physi$\mathrm{cal}^{7}{ }^{\text {and face-to-face social }}{ }^{5}$ pursuits. Additionally, there is the potential for cyberbullying, with a study by the Pew Internet and American Life Project reporting that one in three adolescents who use the Internet have been harrassed online. ${ }^{8}$

While SNS use has grown exponentially in recent years, ${ }^{3}$ particularly among adolescents, ${ }^{5}$ there is still a sizeable number of teenagers who do not use SNSs. ${ }^{3}$ Much of the research to date examining adolescents and SNSs has focused on the benefits and costs associated with using this technol- ogy. However, one aspect of the SNS trend that has received significantly less attention involves the reasons why adolescents choose not to use SNSs. To the best of our knowledge, there is only one study to date that has specifically examined the reasons for non-use. ${ }^{9}$ Participants were American college students, and results showed that non-users identified disinterest, lack of understanding of the appeal of SNSs, and a dislike of engaging in presentation of the self through these sites as reasons for not engaging in SNS use. However, the current study is, to the best of our knowledge, the first to examine the reasons that Australian adolescents give for not engaging in SNS use. Potential benefits of such information may include aspects such as developing policies to respond appropriately to teens' concerns regarding SNSs. This information is likely to be of interest to various groups, including academics, parents, and policy makers, such as schools and governments. Further, given the increasing popularity of SNSs, many companies are now seeing SNSs as a viable channel to approach consumers. ${ }^{10}$ This information is likely, therefore, to be of interest to businesses wanting to adopt appropriate marketing strategies to target this age group. Thus the purpose of the present research was to explore the reasons that adolescents offer for not engaging in SNS use. Given the limited research conducted in this area to date, a qualitative investigation was deemed appropriate.

\section{Method \\ Participants}

As part of a larger study examining attitudes towards SNSs among a sample of Year 9 and 10 Australian secondary school

School of Psychology and Counselling, Queensland University of Technology, Queensland, Australia. 
students, 69 out of 229 students reported that they did not currently use SNSs and were, therefore, eligible for the present qualitative study investigating reasons underlying non-use. Students aged 13 to 18 years $(M=14.64$ years, $S D=1.25$; 40 out of 98 males, 29 out of 131 females) completed the study's questionnaire. Five participants reported having used MySpace in the past, one participant had previously used both MySpace and Facebook, while the remainder of the participants identified that they had not used any SNSs before. All participants provided their reasons for not using SNSs.

\section{Procedure}

Two independent (i.e., non-government) co-educational schools located in middle-class socioeconomic areas agreed to provide their students with the opportunity to participate in the study using established ethical procedures for data collection. While parental permission was required prior to student participation in one of the schools, the second school had a blanket consent policy in place, with the principal consenting to student participation on behalf of the parents. At both schools, completion of the questionnaires was considered students' consent to participate. In all cases, verbal and written instructions were given to all participants, and participants completed the paper questionnaires at their own pace and in selected class times. Participants initially responded to closed-ended questions eliciting demographic information. A definition of online SNSs was then provided for participants:

Online SNSs (e.g., MySpace and Facebook) allow people to create online profiles about themselves and then communicate with others and form online networks of friends. They also act as online diaries. Members are able to chat with each other online, share photos and videos, and also post comments in online forums, blogs, or discussion groups. Online social networking, for this survey, does not refer to e-mail or instant messaging (e.g., MSN). ${ }^{1,11}$

If participants indicated that they did not use a SNS, they were instructed to complete an open-ended item stating: "We would like for you to share with us the reasons why you do not use online social networking sites," with six lines provided for students to handwrite their responses, which was considered adequate space for participants to identify several responses without being overwhelmed by too much space to fill.

\section{Data analysis}

Overall, a conceptual qualitative analysis ${ }^{12}$ of the data was undertaken, which borrowed, in part, from the processes and methods of a grounded theory (GT) approach. ${ }^{13}$ For instance, both authors commenced with a broad search through the data for possible codes (i.e., consistent with GT's open coding). Transcripts were coded by grouping similar responses (i.e., beliefs) into themes for non-use that had been commonly stated by participants. Specifically, both authors (serving as the coders) analyzed the data for beliefs relating to reasons for not using SNSs. Then, consistent with axial coding of responses, the analysis was undertaken, by both coders, in terms of first identifying the beliefs relating to reasons for not using SNSs and subsequently coding each belief in terms of its similarity or dissimilarity to previous beliefs that had been coded (akin to the GT process of constant comparison). Each coder then grouped the similar beliefs together and assigned each group of beliefs an overarching theme. To heighten data trustworthiness, both authors coded the data independently of the other and then came together to discuss their respective findings in relation to both the identification of overarching themes and the grouping of individual beliefs into themes. Overall, there were only a small number of discrepancies between the two coders as part of this process. These discrepancies were subsequently discussed further by the two coders, and themes were only established and beliefs were only classified into a particular theme once the two coders reached consensus. Thus a strength of this approach, in having two coders analyze all of the data separately and then working together to review each other's coding of all of the data, was to ensure a thorough analysis of the data, which functioned to heighten the trustworthiness of the analysis. The findings reported are therefore believed to be an accurate and complete reflection of the responses received.

The findings of the qualitative content analysis ${ }^{12}$ reported below represent the beliefs that participants most commonly reported. Frequencies for a belief $(n)$ are the total number of times a belief was written across all participants. If the same belief was reported multiple times by a single participant, it was only counted once. Given that a small number of participants stated that they were former users of SNSs, the comments elicited from these participants are highlighted in the sections below to account for any discrepancies in reasoning from participants who have never used the technology.

\section{Findings}

\section{Primary reasons}

Many students nominated reasons such as lack of motivation, poor use of time, preference for other forms of communication, preference for other activities, cybersafety concerns, and a dislike of self-presentation online to explain why they did not currently use SNSs.

Lack of motivation. The most common reason stated for non-use of SNSs was no interest or motivation to use them (51\%; 24 males, 11 females). For example, one participant reported, "I don't see the point of using them. They are not fun, entertaining or amusing in any way" (male, 14 years), while another identified, "Can't be bothered setting up a profile" (male, 15 years).

Poor use of time. Other students identified that SNS use was too time-consuming or detracted from time that they could be spending on alternate activities (42\%; 9 males, 20 females). For example, one participant reported, "I don't have time to use these sites. If I do start using these sites, it will take over time that would usually be [spent] studying" (female, 17 years), while another identified that it would be "Too much of a distraction [and] time-consuming" (female, 16 years). All six of the respondents who had previously used SNSs reported that SNS use was too time-consuming and distracting.

Preference for other forms of communication. Some students preferred to use other forms of communication and/or technologies instead of using SNSs (41\%; 16 males, 12 females). 
One participant reported, "I prefer talking to people via phone or face-to-face. I have an e-mail address but that's it" (female, 14 years). Similarly, another student said, "MSN is good enough for me" (male, 13 years). One of the six respondents who had previously used SNSs reported a preference for alternate forms of communication.

Preference for engaging in other activities. Other students identified that they had other activities, interests, or commitments that they preferred or were required to engage in (25\%; 10 males, 7 females). Examples of responses include, "I'd rather read, watch Lost [TV show], or watch X-Files [TV show]" (female, 15 years), and "I don't have time to use such sites because of my sporting commitments" (male, 15 years). One of the six respondents who had previously used SNSs reported a preference for engaging in other activities.

Cybersafety concerns. Some students reported concerns about their cybersafety as a deterrent from using SNSs (23\%; 4 males, 12 females). One student identified, "I stopped using MSN because I was cyberbullied. I [now] use none of these Web sites" (female, 14 years); another said, "[I] don't feel comfortable with having photos up on the Internet for people to comment [on]" (female, 17 years).

Dislike of self-presentation online. Students identified reasons related to the public nature of SNSs and associated self-presentation issues (15\%; 4 males, 6 females) such as, "I hate the idea of rating all my friends or myself being rated against my friends" (female, 13 years), and "I don't have many friends in the real world so there is no point trying to make them online" (male, 14 years) as reasons for not using SNSs.

\section{Secondary reasons}

A few students nominated reasons such as limited access, parents' concerns, and friends' influence as reasons for not currently using SNSs.

Limited access. Some participants reported that limited or no access to the Internet $(10 \% ; 7$ males, 0 females) prevented them from using SNSs. For example, one student said, "We don't have enough monthly Internet usage at home" (male, 13 years), while another identified "I don't have access to the Internet" (male, 15 years).

Parents' concerns. Parental concerns were cited as another reason for not using SNSs by a few participants $(6 \% ; 1$ male, 3 females), with comments such as "My mum doesn't support it" (male, 14 years), and "My parents say it is unsafe, that there is a great amount of Internet bullying" (female, 15 years).

Friends' influence. A couple of other students reported that, "None of my friends have profiles on social networking sites" (male, 13 years) and "None of my friends use it" (male, 14 years) as reasons for non-use of SNSs (4\%; 2 males, 1 female).

\section{Discussion}

This study comprised a preliminary investigation to explore the reasons why Australian adolescents choose not to engage in online social networking. Using qualitative content analysis, the findings revealed a number of common themes among this cohort, and the primary reasons nominated were: lack of motivation, poor use of time, preference for other forms of communication preference for engaging in other activities, cybersafety concerns, and a dislike of selfpresentation online.

The identification of these primary themes suggest a range of reasons for non-use, with specific emphasis on a lack of motivation to engage in SNS use, as well as a poor use of time, by a subsample of adolescents. These major reasons suggest the failure of SNSs to capture the imagination and attention of these adolescents and are consistent with the study that examined reasons for non-use among American college students. ${ }^{9}$ For the small sample of previous SNS users, these reasons were the most cited for not continuing their use of the technology. Interestingly, in the other major themes elicited, there was a stated preference for both other forms of communication and choice of other activities rather than SNSs for participants, suggesting that the medium does not have universal appeal despite its popularity, and that the costs of this form, over other forms of communication, are recognized by some adolescents. Concerns about both cybersafety and fear of ridicule from the public nature of the medium were recognized a number of times and appear to be an important factor in determining non-use for some adolescents.

For the reasons for non-use nominated less often by adolescents, the issue of access suggests that the physical or financial ability to connect to the technology is not a barrier for many of the participants in the current study. Further, the influence of others (both parents and friends) as an explanation for non-use was raised less often than other reasons, and points to more personal rather than socially based reasoning in the decision not to use SNSs. It should be noted that the subsample of participants who had used SNSs before raised issues related to time wasting and preference for other communication forms and activities for their current non-use rather than the other reasons raised by previous non-users such as cybersafety or other concerns related to self-presentation online, limited access to the technology, or the influences of parents or friends.

Overall, these findings highlight that these identified disadvantages to SNS use would require a multipronged approach for site operators in their attempts to appeal to a wider teenage audience. Strategies for more engagement of the teenage market would include the notions that SNS use can help to save time (e.g., interactions with friends but without the time taken to physically meet allows more time for studying and other activities) and that there are reliable mechanisms available to ensure the cybersafety of users (e.g., by programs that monitor and act on any reports of cyberbullying or stalking).

While this is, to the best of our knowledge, one of the first studies to investigate the reasons that adolescents offer for not using SNSs, some limitations are evident. Given that the study's sample consisted of students from two high school year levels only, further investigation using a broader range of ages would provide a more comprehensive perspective of the reasons that adolescents of varying ages may have for not engaging in SNS use. In addition, it would be useful to obtain information about non-users' digital media literacy, access to the Internet, and whether their friends use SNSs to 
understand better the contextual factors contributing to nonuse. Further, given that all participants were from independent schools, it may be useful to investigate further adolescent non-use of SNSs among students from different types of school environments, especially to broaden the socioeconomic status range where Internet access reasons may be more prominent. While this study had the benefit of anonymity by the use of written responses, future research in the area may gain a richer analysis of the phenomenon by undertaking interviews and focus groups to explore in greater depth adolescents' feelings and perceptions about their decision not to use SNSs.

In conclusion, this study showed that lack of motivation, poor use of time, preference for other forms of communication and engaging in other activities, cybersafety concerns, and a dislike of self-presentation online are the major reasons that Australian adolescents offer for not using SNSs. Based on these initial findings, future research in this area will advance our current understanding of this new and growing communication phenomenon, including the perceptions of those adolescents to whom the medium is not proving to be either appealing or necessary for connecting with others.

\section{Acknowledgments}

The authors would like to thank Dr. Shari Walsh for her assistance in the design of the study and Dr. Ioni Lewis for her assistance with the data analysis.

\section{Disclosure Statement}

No competing financial interests exist.

\section{References}

1. Boyd DM, Ellison NB. Social network sites: Definition, history, and scholarship. Journal of Computer-Mediated Communication 2008; 13:210-30.

2. Comscore. (2007) Social networking goes global. www .comscore.com/press / release.asp?press $=1555 \quad$ (accessed Jan. 5, 2009).

3. Lenhart A, Madden M. (2007) Social networking websites and teens: An overview. www.pewinternet.org/ /media//
Files/Reports/2007/PIP_SNS_Data_Memo_Jan_2007.pdf.pdf (accessed Dec. 20, 2008).

4. Nielsen/Netratings. (2008) Pacific surfers ride social networking boom. www.nielsen-online.com/pr/pr_080211_ AU.pdf (accessed Jan. 5, 2009).

5. Livingstone S. Taking risky opportunities in youthful content creation: Teenagers' use of social networking sites for intimacy, privacy and self-expression. New Media \& Society 2008; 10:393-411.

6. Sharif I, Sargent JD. Association between television, movie, and video game exposure and school performance. Pediatrics 2006; 118:1061-70.

7. Vandewater EA, Shim M, Caplovitz AG. Linking obesity and activity level with children's television and video game use. Journal of Adolescence 2004; 27:71-85.

8. Lenhart A. (2007) Cyberbullying and online teens. www .pewinternet.org/ /media/Files/Reports / 2007/PIP\%20 Cyberbullying\%20Memo.pdf.pdf (accessed Dec. 20, 2008).

9. Tufekci Z. Grooming, gossip, Facebook and MySpace: What can we learn about these sites from those who won't assimilate? Information, Communication \& Society 2008; 11: 544-64.

10. Burns, E. (2007) Marketing to social networking sites: Targeted. www.clickz.com/3625536 (accessed July 5, 2010).

11. Hinduja S, Patchin JW. Personal information of adolescents in the Internet: A quantitative analysis of MySpace. Journal of Adolescence 2008; 31:125-46.

12. Joffe H, Yardley L. (2004) Content and thematic analysis. In Marks DF, Yardley L, eds. Research methods for clinical health psychology. London: Sage, pp. 56-68.

13. Draucker CB, Martsolf DS, Ross R, et al. Theoretical sampling and category development in grounded theory. Qualitative Health Research 2007; 17:1137-48.

Address correspondence to: Dr. Katherine White School of Psychology and Counselling Queensland University of Technology Victoria Park Road Kelvin Grove, Qld., 4059 Australia

E-mail: km.white@qut.edu.au 